\title{
Regarding "Neurovascular Manifestations of Hereditary Hemorrhagic Telangiectasia: A Consecutive Series of 376 Patients during 15 Years"
}

W e would like to thank the authors Brinjikji et $\mathrm{al}^{1}$ for their study titled "Neurovascular Manifestations of Hereditary Hemorrhagic Telangiectasia: A Consecutive Series of 376 Patients during 15 Years.” The authors have nicely described the spectrum of cerebrovascular lesions in a large series of patients with hereditary hemorrhagic telangiectasia (HHT).

The authors included only patients with definite HHT (met $\geq 3$ of 4 Curacao criteria) and had a group of 376 patients over a 15 -year period. Could the authors tell us how many patients in total were imaged for screening for HHT during this time period at their institution? Did these patients have any neurologic symptoms, or was neuroimaging performed to screen for brain vascular malformations? The authors stated that screening MR imaging may be warranted for both adults and children. However, the group formulating the guidelines found only Level 3 evidence for this recommendation (opinions of respected authorities, based on clinical experience, descriptive studies, or reports of expert committees). ${ }^{2}$ To better understand the utility of screening, answers to a few questions would be helpful.

Five patients in this study with single nidal brain AVMs presented with hemorrhage. Could the authors describe how many other patients included in the study had hemorrhage over the course of the study (assuming follow-up was available) and how many patients were treated? It would also help if the authors could mention the criteria used for treatment of nidal AVMs in these patients. Did any of the treated patients develop complications from treatment or have hemorrhage on follow-up?

We would also appreciate if the authors could comment on the utility of vascular imaging in this patient subset. CTA/MRA was performed in 140 patients (37.2\%), and DSA performed in 46

http://dx.doi.org/10.3174/ajnr.A4986 patients. The authors acknowledged absence of cerebral angiography in all patients as a limitation of the study because angiography is more sensitive for detection of smaller AVMs. Do the authors advocate angiography in all patients? Other than finding additional small AVMs, could the authors elaborate on the added utility of performing angiography? Did any patients receive repeat imaging?

Of the spectrum of vascular lesions described in patients with HHT, the nidus-type AVMs may not have a completely benign natural history. The rationale for screening patients with HHT for brain vascular malformations has been stated to be to detect a vascular malformation before the development of a debilitating hemorrhage. ${ }^{2}$ However, there is absence of literature proving that screening or preemptive treatment are effective strategies in HHT. It would be very helpful if the authors, through their large series, can answer these questions.

Disclosures: Kimberly Seifert—UNRELATED: Employment: Virginia Commonwealth University, Yale, Comments: residency training compensation.

\section{REFERENCES}

1. Brinjikji W, Iyer VN, Yamaki V, et al. Neurovascular manifestations of hereditary hemorrhagic telangiectasia: a consecutive series of 376 patients during 15 years. AJNR Am J Neuroradiol 2016;37:1479-86 CrossRef Medline

2. Faughnan ME, Palda VA, Garcia-Tsao G, et al. International guidelines for the diagnosis and management of hereditary haemorrhagic telangiectasia. J Med Genet 2011;48:73-87 CrossRef Medline

(D) A. Malhotra

(1D) X. Wu

(D) K. Seifert

Department of Radiology and Biomedical Imaging Yale School of Medicine New Haven, Connecticut 